\title{
Chronos Mobi: uma aplicação móvel multiplataforma para o gerenciamento de projetos
}

\author{
Marcelo Kasperbauer ${ }^{1}$ \\ João Tavares ${ }^{1}$ \\ Marcelo Batista ${ }^{1}$ \\ Alexsandro Filippetto ${ }^{1}$ \\ Giovane Barcelos ${ }^{1}$ \\ Clóvis Silveira ${ }^{1}$ \\ Jorge Barbosa ${ }^{2}$
}

\begin{abstract}
Resumo: Este trabalho propõe uma aplicação multiplataforma para dispositivos móveis voltada ao gerenciamento de projetos. O Chronos Mobi baseia-se no modelo Chronos, cujos componentes englobam mapas mentais, PMBoK e Scrum. A implementação baseou-se no uso do framework Phonegap que prove inúmeros recursos para o desenvolvimento móvel. Para a avaliação do Chronos Mobi realizou-se uma análise comparativa do desempenho da aplicação executando em tablets e smartphones nas plataformas Android, Windows Phone e Symbian.
\end{abstract}

Palavras-chave: Chronos. Computação móvel. Dotproject. Gerenciamento de projetos. Multiplataforma. Phonegap.

\begin{abstract}
This paper proposes a multiplatform application for mobile devices focused on project management. The Chronos Mobi is based on Chronos model, whose components encompass mind maps, PMBoK and Scrum. The implementation was based on the use of Phonegap framework that provides numerous features for mobile development. For the evaluation of Chronos Mobi we conduct a comparative analysis of application performance running on tablets and smartphones on the Android, Windows Phone and Symbiam platforms.
\end{abstract}

Keywords: Chronos. Dotproject. Mobile computing. Multiplatform. Phonegap. Project Management.

\section{Introdução}

Atualmente algumas empresas possuem suas sedes e colaboradores alocados em diferentes localizações, dessa forma, além dos tradicionais conceitos de escopo, preço, tempo e qualidade, as equipes de gerenciamento de projetos devem considerar os efeitos causados pelas diferenças entre países, tais como fuso horário, feriados, reuniões à distância e políticas locais [25].

Observou-se, portanto, a necessidade de uma solução que cubra todo o ciclo de gerenciamento de projetos que seja baseada em patterns consolidados, atuando de forma proativa no gerenciamento de projetos [9]. Constatando-se essa demanda, o presente trabalho tem como objetivo desenvolver uma solução para dispositivos móveis, multiplataforma, seguindo o Modelo Chronos, a fim de aumentar a produtividade e a eficiência no gerenciamento de projetos.

\footnotetext{
${ }^{1}$ Curso de Sistemas de Informação - Facensa, Av. Dr. José Loureiro da Silva, 1991 - Gravataí (RS) - Brasil \{master.kasperbauer@gmail.com\} \{joao, marcelo, alexsandro, giovane, clovis@professor.fac ensa.edu.br\}

${ }^{2}$ Programa Interdisciplinar de Pós-Graduação em Computação Aplicada - Unisinos, Av. Unisinos, 950 - São Leopoldo (RS)

- Brasil

\{jbarbosa@unisinos.br\}
}

http://dx.doi.org/10.5335/rbca.2013.2774 
Do ponto de vista técnico, atualmente um dos principais obstáculos ao se desenvolver aplicativos para dispositivos móveis é o número de diferentes aparelhos e plataformas que devem ser suportadas [20]. Esse obstáculo tem dado origem a frameworks para o desenvolvimento móvel tais como Phonegap [23], rhomobile [28] e titanium [1], que suportam o desenvolvimento para múltiplas plataformas.

Esses frameworks permitem ao desenvolvedor utilizar tecnologias web, tais como HTML5, CSS3, Java script [32] e jquery [19], as quais possuem maior destaque entre o interesse dos desenvolvedores, com 79\% desses declarando que pretendem integrar essas tecnologias aos seus apps, segundo pesquisa realizada entre desenvolvedores para plataformas móveis [19].

Diante desses fatos, o presente trabalho apresenta uma solução multiplataforma, chamada Chronos Mobi, projetada para possibilitar o gerenciamento de projetos utilizando-se dispositivos móveis. O Chronos Mobi foi desenvolvido com o uso do framework Phonegap [23], seguindo o modelo Chronos [9]. A avaliação da solução foi realizada com o uso de smartphones utilizando as plataformas Android, Symbian e Windows Phone, com o intuito de avaliar o tempo gasto pela aplicação para o consumo de serviços web e renderização de suas telas.

Este artigo estrutura-se em oito seções. Na segunda seção são paresentados os trabalhos relacionados. A terceira seção aborda os estudos sobre frameworks voltados para o desenvolvimento móvel. A quarta apresenta conceitos relacionados ao gerenciamento de projetos e a quinta o modelo Chronos e o Chronos Mobi. Na sexta seção são detalhados os experimentos realizados e na sétima seção são apresentados os resultados da avaliação. Por fim, na oitava seção discutem-se as conclusões finais e trabalhos futuros.

\section{Trabalhos relacionados}

Dentre os trabalhos pesquisados, destacam-se as soluções Location Based Task Reminder [14], Task Aware Universal [3], Any.do [14] e Astrid [14] como ferramentas para o gerenciamento de tarefas além das soluções Ap4 Project Manager [14] e Project Management Software [3] como ferramentas para o gerenciamento de projetos.

A Tabela 1 apresenta um comparativo entre os trabalhos relacionados.

Tabela 1: Comparativo entre trabalhos relacionados

\begin{tabular}{|c|c|c|c|c|}
\hline Solução & $\begin{array}{l}\text { Sensibilidade ao } \\
\text { Contexto }\end{array}$ & $\begin{array}{l}\text { Gerenciamento de } \\
\text { Perfil de Usuário }\end{array}$ & Multiplataforma & Função Principal \\
\hline $\begin{array}{l}\text { Location Based Task } \\
\text { Reminder }\end{array}$ & $\begin{array}{c}\text { Apenas } \\
\text { localização }\end{array}$ & Não & $\begin{array}{l}\text { Não, apenas } \\
\text { Android }\end{array}$ & $\begin{array}{c}\text { Gerenciamento de } \\
\text { tarefas }\end{array}$ \\
\hline $\begin{array}{l}\text { Task Aware } \\
\text { Universal }\end{array}$ & $\begin{array}{c}\text { Apenas } \\
\text { localização }\end{array}$ & Apenas ajuste de tela & Não, apenas $i O S$ & $\begin{array}{l}\text { Gerenciamento de } \\
\text { tarefas }\end{array}$ \\
\hline Ap4 Project Manager & Não & Não & $\begin{array}{l}\text { Não, apenas } \\
\text { Android }\end{array}$ & $\begin{array}{l}\text { Gerenciamento de } \\
\text { projetos }\end{array}$ \\
\hline $\begin{array}{l}\text { Project Management } \\
\text { Software }\end{array}$ & Não & Não & Não, apenas $i O S$ & $\begin{array}{l}\text { Gerenciamento de } \\
\text { projetos }\end{array}$ \\
\hline Astrid & $\begin{array}{c}\text { Apenas } \\
\text { localização }\end{array}$ & Múltiplos usuários & $\begin{array}{l}\text { Não, apenas } \\
\text { Android }\end{array}$ & $\begin{array}{l}\text { Gerenciamento de } \\
\text { tarefas }\end{array}$ \\
\hline Any.do & Não & Não & $\begin{array}{l}\text { Não, apenas } \\
\text { Android }\end{array}$ & $\begin{array}{l}\text { Gerenciamento de } \\
\text { tarefas }\end{array}$ \\
\hline Chronos Mobi & $\begin{array}{c}\text { Sim, localização, } \\
\text { dispositivo e } \\
\text { conexão }\end{array}$ & $\begin{array}{l}\text { Sim, ajuste de tela e } \\
\text { perfil de usuário }\end{array}$ & $\begin{array}{l}\text { Sim, Android, } \\
\text { Windows Phone, } \\
\text { iOS, Symbian, } \\
\text { Blackberry e Bada }\end{array}$ & $\begin{array}{l}\text { Gerenciamento de } \\
\text { projetos }\end{array}$ \\
\hline
\end{tabular}

Revista Brasileira de Computação Aplicada (ISSN 2176-6649), Passo Fundo, v. 5, n. 1, p. 84-97, abr. 201385 
O quesito "sensibilidade ao contexto" informa que a solução utiliza informações relativas ao contexto no qual encontra-se o usuário. O "gerenciamento de perfil de usuário" mostra que a solução é capaz de adaptar-se de acordo com o perfil do usuário. O quesito "multiplataforma" indica que a solução foi projetada para executar em múltiplas plataformas de software. O item "função principal” tem o intuito de demonstrar o objetivo principal da solução.

Conforme observado na comparação acima, o Chronos Mobi possui como diferenciais a capacidade de gerenciamento de contexto e perfil de usuário, funcionamento multiplataforma e em diferentes dispositivos, proporcionando uma aplicação móvel para o gerenciamento de projetos.

\section{Desenvolvimento móvel}

Uma nova abordagem para o desenvolvimento de aplicativos para dispositivos móveis tem sido aplicada através do uso de tecnologias web tais como HTML5 e de frameworks específicos, que funcionam como drives que são capazes de converter a linguagem web para a linguagem nativa da plataforma [20].

Desenvolver aplicativos utilizando frameworks como Phonegap [23], rhomobile [28] e titanium [1], agregam a vantagem do desenvolvimento de um único código genérico para múltiplas plataformas distintas, eliminando a necessidade de conhecimento e compilação em linguagens nativas de cada sistema [23].

\subsection{Framework Phonegap}

Phonegap é um framework open source, para o desenvolvimento móvel. O Phonegap oferece ao desenvolvedor a possibilidade de trabalhar com múltiplas plataformas, tais como, iOS, Android, Blackberry, Windows Phone, WebOS e Symbian [23]. Esse framework possibilita o desenvolvimento para dispositivos móveis de forma transparente com o uso de tecnologias web, tais como, HTML5, CSS3, Java Script [32], Prototype [27] e Jquery [19], sem a necessidade de compilar seus aplicativos para múltiplas plataformas [21].

A Figura 1 apresenta o funcionamento do Phonegap onde o código web é processado pelo framework que responsabiliza-se pelo deploy para as múltiplas plataformas [23], fazendo a conversão da linguagem web para a linguagem nativa da plataforma.

A API deste framework é composta de recursos para o desenvolvimento móvel para múltiplas plataformas, tais como Accelerometer, Camera, Capture, Compass, Connection, Contacts, Device, Events, File, Geolocation, Media, Notification e Storage [23]. Ressalta-se que o Chronos Mobi utiliza os recursos de Geolocation, Device e Connection.

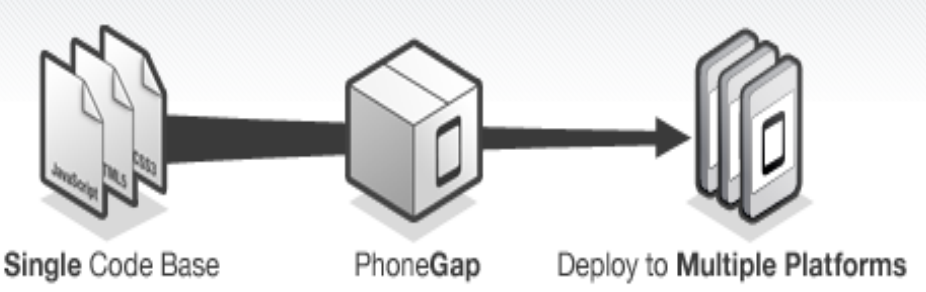

Figura 1: Phonegap geração de código [23]

\subsection{Comparativo entre frameworks}

Dentre os frameworks pesquisados para o desenvolvimento do Chronos Mobi, foram selecionados aqueles que suportam mais de uma plataforma e que aplicam tecnologias web. Destacam-se, portanto, o Phonegap [23], rhomobile [28] e titanium [1].

Conforme observado na Tabela 2, dentre os frameworks pesquisados, o Phonegap é o que oferece suporte ao maior número de plataformas, possuindo mais recursos em sua API e suporte ao maior número de tecnologias web. Devido às vantagens apresentadas pelo Phonegap em relação aos demais frameworks, optou-se por essa solução para o desenvolvimento do Chronos Mobi. 
Tabela 2: Comparativo entre frameworks para mobilidade

\begin{tabular}{|c|c|c|c|}
\hline & Phonegap & Titanium & Rhomobile \\
\hline Multiplataforma & $\begin{array}{c}\text { Sim (Android, iOS, } \\
\text { Symbian, Web OS, } \\
\text { Blackberry e Windows } \\
\text { Phone) }\end{array}$ & $\begin{array}{l}\text { Sim (somente } \\
\text { Android e iOS) }\end{array}$ & $\begin{array}{l}\text { Sim (Android, Windows } \\
\text { Phone e } i O S)\end{array}$ \\
\hline $\begin{array}{l}\text { Tecnologias } \\
\text { Web }\end{array}$ & $\begin{array}{c}\operatorname{Sim}(H T M L, C S S, \text { Java } \\
\text { Script, Jquery, XUI, } \\
\text { Prototype e dojo) }\end{array}$ & $\begin{array}{l}\text { Sim }(H T M L, \text { Java } \\
\text { Script e CSS })\end{array}$ & $\operatorname{Sim}(H T M L)$ \\
\hline $\begin{array}{r}\text { Recursos de } \\
\text { geolocalização }\end{array}$ & $\begin{array}{l}\text { Sim (integrado com GPS } \\
\text { e Geolocation) }\end{array}$ & $\begin{array}{l}\text { Sim (integrado com } \\
G P S)\end{array}$ & Sim (integrado com GPS) \\
\hline $\begin{array}{l}\text { Recursos de } \\
\text { hardware }\end{array}$ & $\begin{array}{c}\text { Sim (controla os } \\
\text { principais recursos do } \\
\text { dispositivo) }\end{array}$ & Não possui & Não possui \\
\hline
\end{tabular}

\subsection{Plataformas, dispositivos e licenças}

Desenvolveu-se o Chronos Mobi com o uso das tecnologias web suportadas pelo Phonegap, dessa forma, o projeto tornou-se apto a ser compilado para múltiplas plataformas. Para a análise realizada neste trabalho, foram selecionadas as plataformas Android, Windows Phone e Symbian.

Android é uma plataforma open source projetada para ser executada em tablets e smartphones. Desenvolvida pela Google, baseia-se em uma versão modificada do Linux. Para executar o aplicativo no dispositivo Android não houve a necessidade de comprar licença de desenvolvedor. A geração da aplicação ocorreu através da IDE eclipse [13] onde o app é exportado para o formato apk, que é o formato suportado pelo Android.

Symbian é uma plataforma open source projetada para executar em smartphones desenvolvida por um conjunto de empresas, como Nokia, Siemens e Panasonic, foi desenvolvido com o intuito de ser um sistema modular, dando a empresas a capacidade de desenvolver a sua própria interface [8]. Para executar o aplicativo no dispositivo Symbian não houve a necessidade de comprar licença de desenvolvedor, a geração da aplicação se deu através do software cygwin [10].

Windows Phone é a plataforma desenvolvida pela Microsoft, projetada para smartphones com o conceito de telas não personalizáveis mantendo sempre o padrão visual da plataforma [22]. Para executar o aplicativo no dispositivo Windows Phone, é necessário efetuar o cadastro de desenvolvedor no App Hub [6] e comprar a licença de desenvolvedor individual que permite utilizar em até três dispositivos. A geração do aplicativo pode ser feita através da IDE Visual Studio [22].

iOS é o sistema operacional desenvolvido pela Apple projetado para os dispositivos, iPhone, iPad e iPod Touch, foi desenvolvido utilizando todo o conhecimento adquirido na criação do Mac OS X [3]. Para executar o aplicativo no dispositivo iOS, alguns passos são necessários, dentre os quais a aquisição de um appleID, que pode ser feita gratuitamente no Member Center [4], seguido da compra da licença de desenvolvedor da Apple. Atualmente existem dois tipos de licença, a standard e a corporativa, ambas podem ser adquiridas no apple developer [5]. Após a compra, é necessário enviar um fax com os dados fornecidos pelo site da Apple e aguardar pela validação do pedido e confirmação da compra. Após a confirmação, a aplicação pode ser gerada utilizando-se um computador Mac e a IDE xcode [34]. Para este trabalho, a plataforma iOS não foi utilizada porque a licença de desenvolvedor não foi disponibilizada tempestivamente.

\section{Fundamentos em gerenciamento de projetos}

Organizações executam trabalho. O trabalho geralmente envolve operações e projetos, estes, por sua vez, compartilham pessoas, recursos e planejamento. O gerenciamento de projetos é a aplicação do conhecimento, habilidades, ferramentas e técnicas para as atividades relacionadas a um projeto [25]. 
O modelo Chronos tem como objetivo principal atuar de forma pró-ativa no gerenciamento de projetos, seguindo as boas práticas sugeridas pelo PMBoK [24], que é um guia que contém as melhores práticas para gestão de projetos, formalizando diversos conceitos no que concerne ao seu gerenciamento. O PMBoK foi proposto pelo Project Management Institute (PMI) [26].

O PMI tem como intuito avançar na prática, na ciência e na área de gerenciamento de projetos em todo o mundo, de uma maneira consciente e pró-ativa, para que as organizações em todos os lugares apoiem, valorizem e utilizem o gerenciamento de projetos - alcançando mais resultados positivos [26].

\section{1 dotProject}

O dotProject é uma aplicação open source para o gerenciamento de projetos, mantida por uma vasta comunidade de desenvolvedores voluntários [12], tendo como objetivo prover um ambiente aberto para o gerenciamento de projetos. Como uma aplicação web, o dotProject foi desenvolvido em PHP com banco de dados MySQL e executando em servidor apache-tomcat [18].

Para o desenvolvimento da camada web do modelo Chronos, situada no servidor do projeto, utilizou-se o dotProject como base. Essa camada armazena a base de dados utilizada pelo Chronos Mobi, com informações relativas a organizações, projetos, tarefas, usuários e geolocalização.

\subsection{Mapas mentais}

Um mapa mental é um diagrama utilizado para a organização e gestão de ideias. Pode ser utilizado como uma poderosa ferramenta de aprendizado, memorização e organização de informações [16].

O modelo Chronos utiliza mapas mentais para a iniciação de projetos, sendo possível organizar as tarefas relativas a projetos, uma vez que os seus elementos são dispostos intuitivamente de acordo com a importância dos conceitos e organizados em ramos.

\subsection{Scrum}

O Scrum apresenta-se como uma metodologia ágil que tem como objetivo definir um processo para projeto, que seja focado nas pessoas [30]. O modelo Chronos aplica o Scrum na fase de execução de projetos, tendo como práticas gerenciais Product Backlogs, que tratam da coleta de requisitos a partir de reuniões entre todos os envolvidos no projeto. O Daily Scrum é uma rápida reunião diária, geralmente de 15 minutos, entre os membros do time no qual são definidas as tarefas do dia e demonstrados os resultados das tarefas anteriores. Já nos Sprints são implementados os itens definidos nos Product Backlogs. Para cada Sprint é realizada uma reunião na qual é feita uma retrospectiva dos últimos acontecidos e planejamento da próxima Sprint [30].

\subsection{Modelo Chronos}

O projeto Chronos vem sendo desenvolvido por pesquisadores do grupo de pesquisa GMobile (Facensa) e do laboratório de computação móvel - Mobilab (Unisinos), tendo por base os conceitos previstos pelo modelo Chronos [9].

O modelo Chronos utiliza mapas mentais para a organização das ideias resultantes do braimstorming seguindo as boas práticas sugeridas pelo $P M B o K$ e Scrum para a execução de tarefas e atividades [9]. Seguindo os conceitos da computação ubíqua, que foi definida por Mark Weiser como "elementos de software e hardware especializados, conectados por redes, ondas de radio e infravermelho, serão tão ubíquos que não será possível notar a sua presença” [33].

O modelo Chronos surgiu a partir da constatação da necessidade de uma solução que cubra todo o ciclo de vida de gerenciamento de projetos, baseando-se no uso de patterns consolidados e atuando de forma pró-ativa no gerenciamento de projetos [9].

Esse modelo cobre todos os aspectos do gerenciamento de projetos com a adoção do paradigma $u$-project. A fase de iniciação (pré-projeto) é realizada utilizando a ferramenta de mapa mental disponível no painel de controle do modelo, onde o brainstorming é registrado diretamente na ferramenta de mapa mental a qual está incorporada no modelo. A fase de planejamento (projeto) adota as melhores práticas sugeridas pelo $P M B o K$, convertendo o mapa mental para Work Breakdown Structure (WBS). Depois disso, os pacotes de tarefas do WBS 
são convertidos em Product Backlogs do Scrum. Na fase de execução, as Sprints do projeto são geradas com os Product Backlogs já criados, alocando automaticamente os stakeholders envolvidos, de acordo com os seus perfis. Ao final, na fase de pós-projeto, relatórios e estatísticas podem ser obtidos para alimentar aplicações de Business Intelligence $(B I)[9]$.

O modelo é dividido em oito componentes, contendo três agentes de software, quatro módulos e o painel de controle, conforme se visualiza na Figura 2.

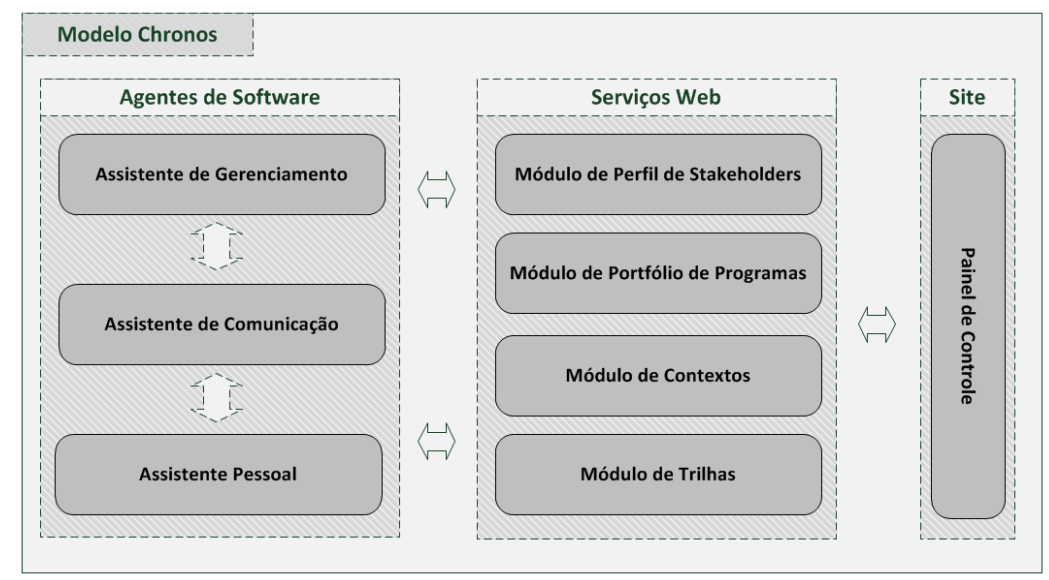

Figura 2: Arquitetura do modelo Chronos

O assistente pessoal (AP) é responsável por monitorar o comportamento do usuário, gerenciar múltiplos perfis de usuário de um mesmo stakeholder e prover artefatos e recursos, seguindo os conceitos da computação ubíqua, onde o perfil de usuário apresentada-se como a síntese das características e preferências do usuário [29].

$\mathrm{O}$ assistente de comunicação (AC) é responsável por trocar mensagens entre o AP e o assistente de gerenciamento (AG), enquanto o AG é responsável por inferir e controlar os módulos do Modelo Chronos, englobando as regras de negócio baseadas nos mapas mentais, Scrum e PMBoK [9]. O módulo chamado perfil de Stakeholder contém a classificação de todos os stakeholders envolvidos. O módulo chamado portfólio de programas contém todos os projetos e programas do usuário.

O módulo de contexto contém a base de armazenamento dos contextos relativos aos projetos e programas. O módulo de Trilhas contém históricos do usuário como, por exemplo, decisões de projetos passados [9]. Esse módulo segue os conceitos de contexto previstos pela computação ubíqua, onde sensibilidade ao contexto envolve toda informação que possa ser utilizada para caracterizar a situação de uma entidade, que, por sua vez pode ser uma pessoa, um lugar ou um objeto que seja considerado relevante para a interação entre o usuário e a aplicação, incluindo o próprio usuário e a aplicação [11].

\subsection{Chronos Mobi}

O Chronos Mobi é uma solução que se concentra no gerenciamento de projetos, tendo como principal objetivo fornecer ao gerente de projetos uma ferramenta que promova produtividade e agilidade com o uso de dispositivos móveis, independentemente da plataforma utilizada.

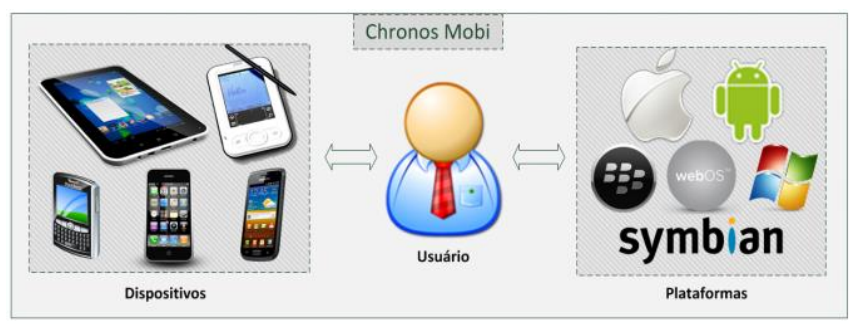

Figura 3: Chronos Mobi: multiplataforma e multidispositivo*

A Figura 3 apresenta o Chronos Mobi, projetado para executar em múltiplas plataformas e múltiplos tipos de dispositivos, dessa forma, o usuário poderá utilizar a mesma em smartphones, tablets e palms que possuam as 
plataformas suportadas pelo framework Phonegap [23], estas são iOS da Apple, Android da Google, Blackberry da Research in Motion, Windows Phone da Microsoft, Web OS da HP, e Symbian da Nokia. Através do gerenciamento de perfil de usuário, essa solução é capaz de adaptar suas telas de acordo com o dispositivo, conexão e perfil do usuário. Através do gerenciamento do contexto o usuário é capaz de gerenciar os projetos e tarefas que constam em seu portfólio e dashboard (painel de visualização), visualizando aqueles que são relativos ao contexto no qual o mesmo está inserido.

A Figura 4 mostra que a solução baseia-se no padrão de projetos Model View Control (MVC), onde na camada model encontram-se a base de dados e a aplicação web dotProject [12] que alimenta a base de dados, com dados relativos a organizações, projetos, tarefas, usuários e geolocalização. Na camada de view encontram-se as telas do Chronos Mobi e as features disponíveis no framework Phonegap, que processa dados relativos ao dispositivo móvel, conexão e localização e envia para a camada control. Essa, por sua vez, é composta de três módulos de gerenciamento de perfil de usuário, de gerenciamento de contexto e de autenticação de usuário. O Chronos Server possui uma camada intermediária que contém o barramento de serviços (Web Services) o qual disponibiliza ao Chronos Mobi funcionalidades, tais como serviços de autenticação, serviço de portfólio e dashboard de projetos e serviços de listagem, atualização e dashboard de tarefas.

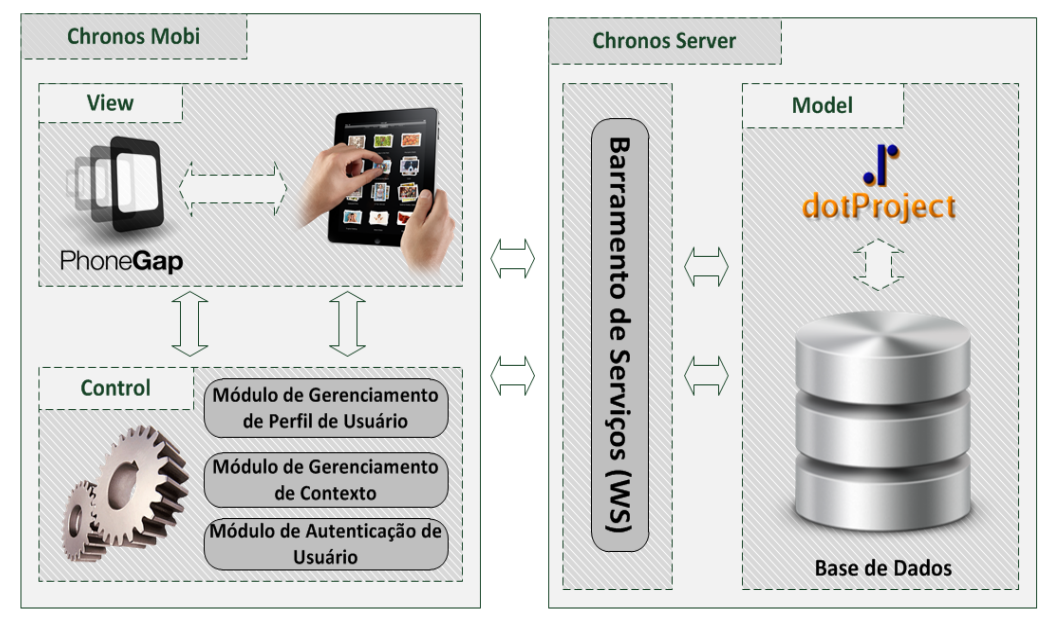

Figura 4: Arquitetura global da solução

A Figura 5 apresenta o diagrama de classes utilizadas pelo Chronos Mobi, estes componentes possuem sua estrutura similar à contida nas tabelas da base de dados do dotProject. Essas classes foram desenvolvidas em linguagem Java e ficam na camada Server da solução.

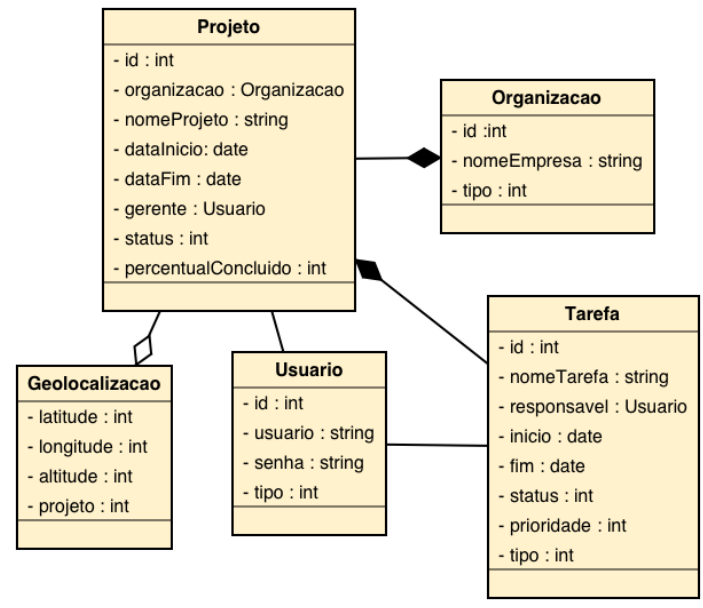

Figura 5: Diagrama de classes do Chronos Mobi

A classe "organização" é utilizada para representar os dados relativos às organizações cadastradas no dotProject; a classe "projeto", representa a lista de projetos que uma organização possui, contendo dados relativos 
ao projeto e um usuário do tipo gerente que é o responsável pelo projeto. A classe "tarefa" representa a lista de tarefas de um projeto, contendo dados relativos à tarefa e usuário responsável. A classe "usuário", representa os tipos de usuário, que podem ser gerente, supervisor ou empregado, além de seus dados de login. A classe "geolocalizacao" é responsável por vincular um projeto a uma localização, contendo dados de latitude, longitude e altitude.

A Figura 6 apresenta o diagrama de sequência que representa a transação dos dados da solução, onde o Phonegap coleta dados de usuário (login) e dados de dispositivo (Device, Geolocation e Connection) através dos recursos disponíveis no Phonegap [23], enviando-os ao Web Service, desenvolvido em linguagem Java e executado em servidor Apache Tomcat, que, por sua vez, se responsabiliza por buscar dados na base de dados do dotProject e retornar os mesmos para o Phonegap no formato JavaScript Object Notation JSON, que é um formato de texto leve e independente de linguagem [20]. O Phonegap é responsável por tratar o JSON para exibição dos dados na tela.

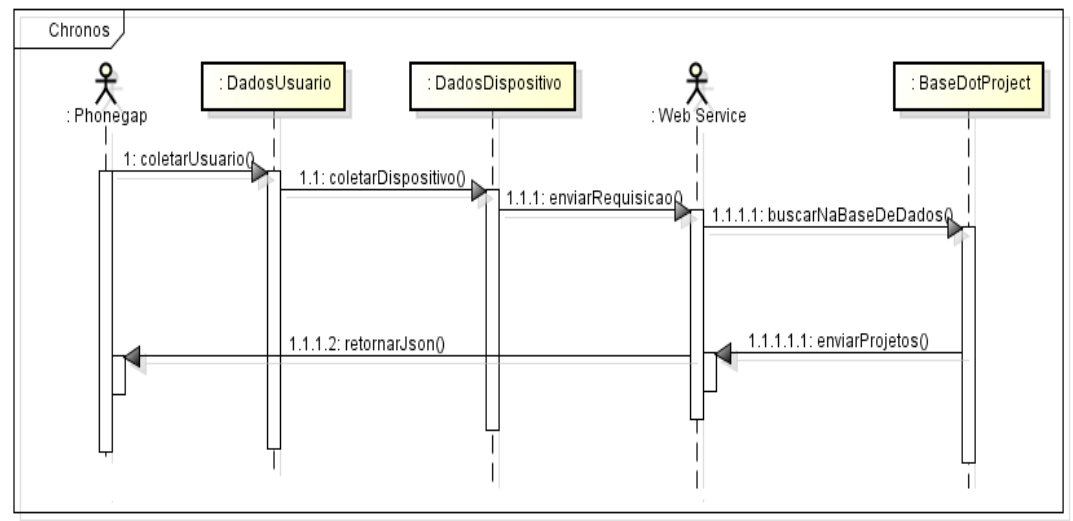

Figura 6: Diagrama de sequência do Chronos Mobi

O módulo de autenticação é responsável por, a partir das informações preenchidas pelo usuário na tela de login, efetuar a autenticação do usuário no Chronos Server. Após isso, o servidor Chronos devolverá os dados de usuário e portfólio de projetos do mesmo de acordo com o seu contexto e perfil.

O módulo de gerenciamento de perfil de usuário é responsável por gerenciar as informações relativas aos projetos e preferências do usuário. Para esse gerenciamento serão utilizados os recursos de Device e Connection do Phonegap. O histórico de preferências do usuário é mantido na base de dados do Chronos e tem como intuito sugerir ao usuário atualizações para suas tarefas de acordo com as ações mais executadas.

O módulo de gerenciamento de contexto é responsável por disponibilizar ao usuário os projetos de seu portfólio e dashboard de acordo com o contexto no qual o usuário está incluído. O usuário tem a possibilidade de associar projetos a um contexto através da edição de projetos contida no dotProject. Após, o projeto será exibido no Chronos Mobi quando o usuário estiver no contexto ao qual o projeto pertence. A localização do dispositivo móvel será obtida através do recurso de Geolocation do Phonegap [23].

\section{Experimentos realizados}

Desenvolveu-se um estudo comparativo a fim de avaliar o desempenho da aplicação em múltiplas plataformas. Definiram-se dois objetivos para a avaliação: (a) avaliar o tempo gasto para a renderização da tela considerando-se o tamanho trafegado; (b) avaliar o tempo gasto para o consumo do serviço web considerando o tamanho trafegado;

A avaliação foi realizada utilizando uma rede WiFi com velocidade de $2 \mathrm{MB}$, comum tanto para o servidor Chronos quanto para os dispositivos móveis. As condições de rede se mantiveram iguais para a avaliação de cada dispositivo.

O servidor Chronos utilizado para a avaliação, possui sistema operacional Windows XP Professional 2002/ Service Pack 3, com 496 MB de memória RAM, processador Intel Core 2 CPU 6300 @ 1.86GHz. O Web Service 
foi executado em servidor de aplicação Apache Tomcat 7, efetuando a integração com os dados persistidos pela versão 2.1.5 do dotProject na base de dados mySQL 5.08.

\subsection{Configuração dos dispositivos móveis}

Foram selecionados para a análise comparativa smartphones de diferentes fabricantes utilizando diferentes plataformas dentre as suportadas pelo Chronos Mobi.

A Tabela 3 apresenta a especificação detalhada destes dispositivos.

Tabela 3: especificação dos dispositivos

\begin{tabular}{clll}
\hline Sistema operacional & \multicolumn{1}{c}{ Android } & Symbiam & Windows phone \\
\hline Versão & 2.1 & OS & 7.5 \\
Memória & $2 \mathrm{~GB}$ & $8 \mathrm{~GB}$ & $8 \mathrm{~GB}$ \\
Processador & ARMv6 600 MHz & ARM 600 MHz & ARM 1.4 GHz \\
Dispositivo & Galaxy Mini & E6 & OMNIA W \\
Fabricante & Samsung & Nokia & Samsung \\
\hline
\end{tabular}

O quesito "versão" informa qual a versão da plataforma avaliada. O quesito "memória" refere-se à capacidade interna do dispositivo. O quesito "processador" informa a capacidade de processamento do dispositivo e tipo de processador. O quesito "dispositivo" informa qual aparelho foi utilizado para a plataforma e, finalmente, o quesito "fabricante" informa a marca do dispositivo.

Conforme se observa nas especificações da Tabela 3, o dispositivo avaliado com maior capacidade de processamento e memória utiliza a plataforma Windows Phone.

\subsection{Preparação dos dados no dotProject}

O dotProject [12] foi alimentado com dados de projetos e tarefas para os testes que foram realizados executando-se em diferentes plataformas. Inicialmente, cadastrou-se a organização FACENSA conforme se visualiza na Figura 7.

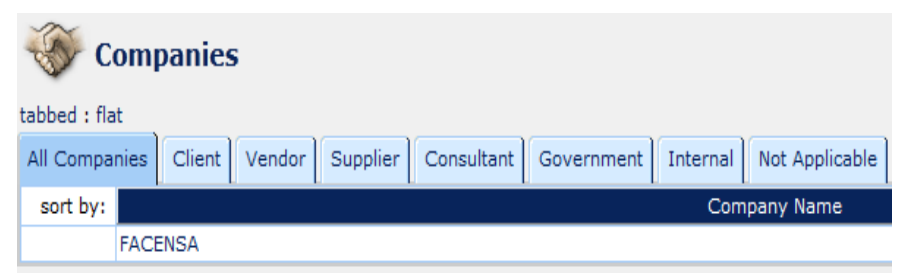

Figura 7: Tela de organizações do dotProject

Foram associados três projetos à organização FACENSA: (a) pesquisa de framework para mobilidade; (b) pesquisa sobre sistemas distribuídos e (c) pesquisa sobre sistemas ubíquos, conforme se visualiza na Figura 8.

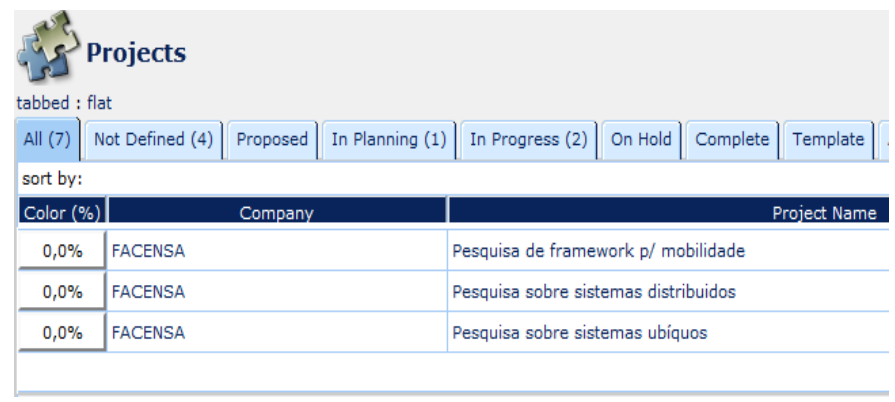

Figura 8: Tela de projetos do dotProject

Cada projeto foi alimentado com três tarefas, o projeto "Pesquisa de framework para mobilidade" teve as seguintes tarefas cadastradas: (a) estudo do framework titanium [1]; (b) estudo do framework rhomobile [28] e (c) estudo do framework Phonegap [23]. O projeto "Pesquisa sobre sistemas distribuídos" foi alimentado com as 
tarefas: (a) estudo sobre sistemas distribuídos; (b) pesquisa de sistemas e (c) comparativo entre sistemas. O projeto "Pesquisa sobre sistemas ubíquos" teve como tarefas cadastradas: (a) pesquisa de sistemas sensíveis ao contexto; (b) pesquisa de sistemas com perfil de usuário e (c) pesquisa de sistemas que consideram smart spaces.

\section{Resultados do experimento}

A Tabela 4 apresenta os resultados obtidos através do objetivo (a) dessa avaliação, utilizando o Chronos Mobi nas plataformas Android, Symbian e Windows Phone, que, por sua vez, utilizam os dispositivos previamente especificados.

Tabela 4: Resultados do objetivo (a)

\begin{tabular}{ccccc}
\hline Tela & $\begin{array}{c}\text { Tamanho Trafegado } \\
(\mathrm{KB})\end{array}$ & $\begin{array}{c}\text { Android } \\
(\text { seg.) }\end{array}$ & $\begin{array}{c}\text { Symbian } \\
(\text { seg.) }\end{array}$ & $\begin{array}{c}\text { Windows Phone } \\
(\text { seg.) }\end{array}$ \\
\hline Autenticação & 2,15 & 6 & 6 & 6 \\
Dashboard de projetos & 9,47 & 8 & 5 & 9 \\
Portfolio & 6,21 & 5 & 3 & 6 \\
Dashboard de tarefas & 9,45 & 8 & 1 & 8 \\
Lista de tarefas & 6,36 & 2 & 1 & 3 \\
\hline
\end{tabular}

Os itens acima se referem ao tempo gasto em segundos por cada plataforma para a renderização do $H T M L$ trafegado considerando o tamanho trafegado para cada ação.

$\mathrm{O}$ item referente à tela de autenticação demonstra que para a renderização do HTML com tamanho de 2,15KB consumiu-se 6 segundos em todas as plataformas. O item referente à tela de Dashboard de projetos demonstra que para a renderização do $H T M L$ de tamanho 9,47KB gastaram-se 8s na plataforma Android, 5s no Symbian e 9s no Windows Phone. O item referente à tela de portfolio demonstra que para a renderização do HTML de tamanho 6,21 KB gastaram-se 5s na plataforma Android, 1s no Symbian e 8s no Windows Phone. O item referente à tela de Dashboard de tarefas demonstra que para a renderização do $H T M L$ de tamanho $9,45 K B$ gastaram-se 8s na plataforma Android, 1s no Symbian e 8s no Windows Phone. O item referente à tela de lista de tarefas demonstra que para a renderização do $H T M L$ de tamanho 6,36KB gastaram-se 2s na plataforma Android, 1s no Symbian e 3s no Windows Phone. A Figura 9 apresenta o gráfico sintetizando os resultados da Tabela 4.

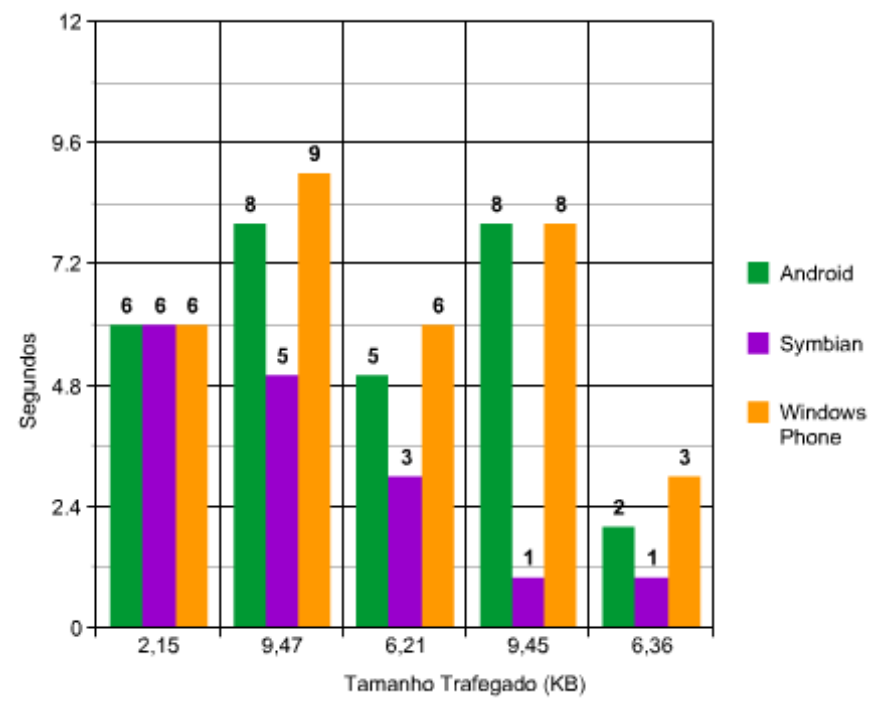

Figura 9: Gráfico de resultados do objetivo (a)

Na Figura 10 é possível observar o HTML que foi renderizado nas telas dashboard de projetos (a), portfólio (b), dashboard de tarefas (c) e lista de tarefas (d).

As setas que constam na imagem têm como objetivo demonstrar o fluxo sequencial de navegação do usuário entre as telas. Após efetuar o login, exibe-se a tela de dashboard de projetos que contêm a lista de projetos gerenciados pelo usuário. Ao clicar no gráfico, a tela é redirecionada para o portfólio de projetos, que contém as 
pastas relativas a estes. Ao selecionar um projeto ele é redirecionado ao dashboard de tarefas, que exibe os resultados pertinentes às tarefas deste. Ao clicar no gráfico, ele é redirecionado para a tela de lista de tarefas, que contém a lista de tarefas, para visualização ou atualização.

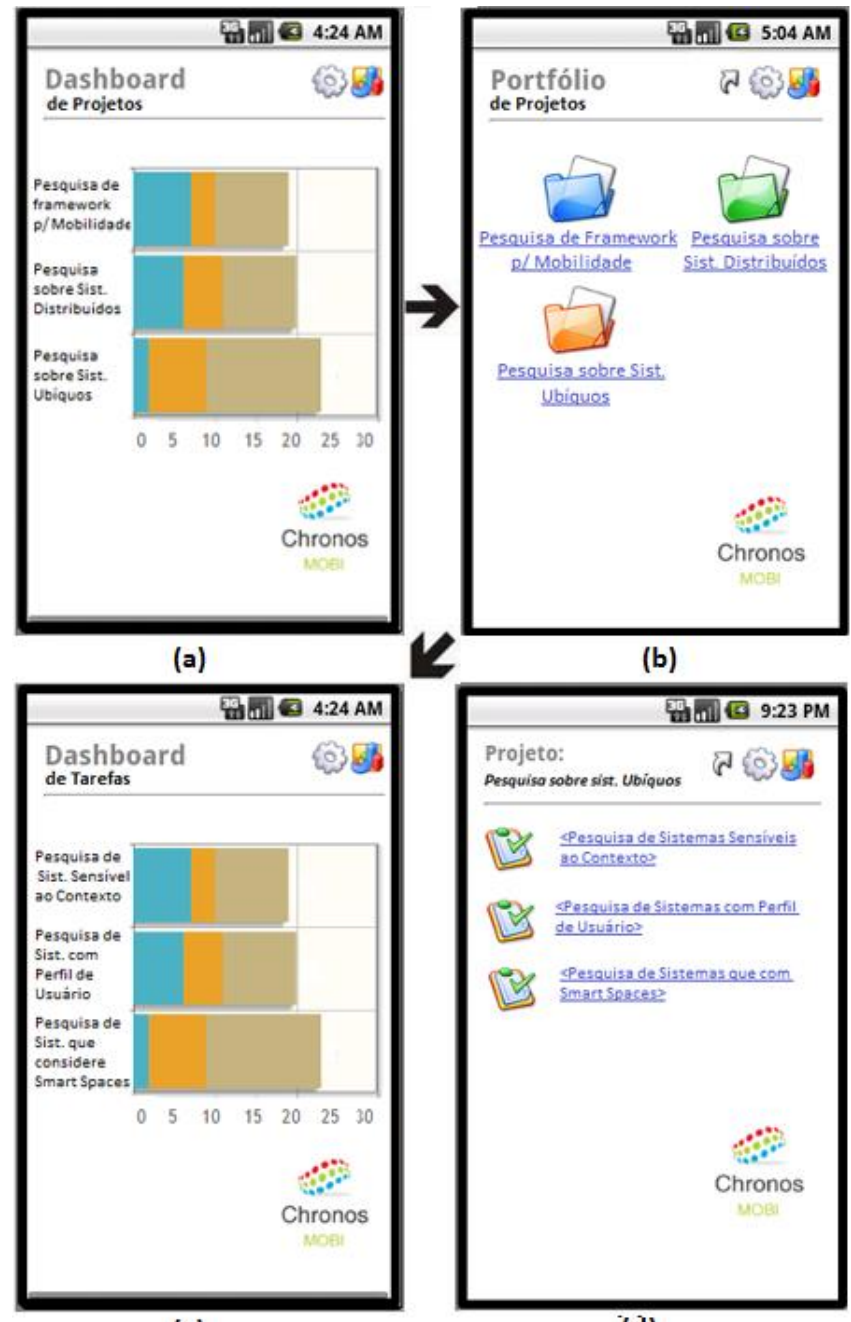

(c)

(d)

Figura 10: Telas do Chronos Mobi:

(a) Dashboard de Projetos (b) Portfólio (c) Dashboard de tarefas (d) Lista de tarefas

A Tabela 5 apresenta os resultados obtidos para o objetivo (b) desta avaliação, utilizando o Chronos Mobi nas plataformas Android, Symbian e Windows Phone, utilizando os dispositivos especificados na Tabela 3.

Tabela 5: Resultados do objetivo (b)

\begin{tabular}{ccccc}
\hline Tela & $\begin{array}{c}\text { Tamanho Trafegado } \\
(\text { KB) }\end{array}$ & $\begin{array}{c}\text { Android } \\
\text { (seg.) }\end{array}$ & $\begin{array}{c}\text { Symbian } \\
\text { (seg.) }\end{array}$ & $\begin{array}{c}\text { Windows Phone } \\
\text { (seg.) }\end{array}$ \\
\hline Autenticação & 0,05 & 4 & 3 & 4 \\
Dashboard de projetos & 0,22 & 6 & 4 & 6 \\
Portfolio & 1,03 & 4 & 2 & 5 \\
Dashboard de tarefas & 0,24 & 5 & 2 & 5 \\
Lista de tarefas & 1,02 & 4 & 2 & 4 \\
\hline
\end{tabular}

Os itens acima se referem ao tempo gasto em segundos por cada plataforma para o consumo do serviço web considerando o tamanho trafegado para cada ação. 
O item referente ao consumo do serviço de autenticação demonstra o tempo de requisição/resposta co conteúdo trafegado de $0,05 \mathrm{~KB}$, tendo sido gastos $4 \mathrm{~s}$ nas plataformas Android e Windows Phone e $3 \mathrm{~s}$ no Symbian. O item referente ao consumo do serviço de Dashboard de Projetos demonstra o tempo de requisição/resposta com conteúdo trafegado de $0,22 \mathrm{~KB}$, tendo sido gastos 6 s nas plataformas Android e Windows Phone e 4s no Symbian. $\mathrm{O}$ item referente ao consumo do serviço de portfólio de projetos demonstra o tempo de requisição/resposta com conteúdo trafegado de $1,03 \mathrm{~KB}$, tendo sido necessários $4 \mathrm{~s}$ na plataforma Android, $2 \mathrm{~s}$ no Symbian e $5 \mathrm{~s}$ no Windows Phone. O item referente ao consumo do serviço de Dashboard de tarefas demonstra o tempo de requisição/resposta com conteúdo trafegado de $0,24 K B$, que exigiu 5 s nas plataformas Android e Windows Phone e 2 s no Symbian. O item referente ao consumo do serviço de lista de tarefas demonstra o tempo de requisição/resposta com conteúdo trafegado de $1,02 \mathrm{~KB}$, no qual foram gastos $4 \mathrm{~s}$ nas plataformas Android e Windows Phone e 2s no Symbian. A Figura 11 apresenta o gráfico sintetizando esses resultados.

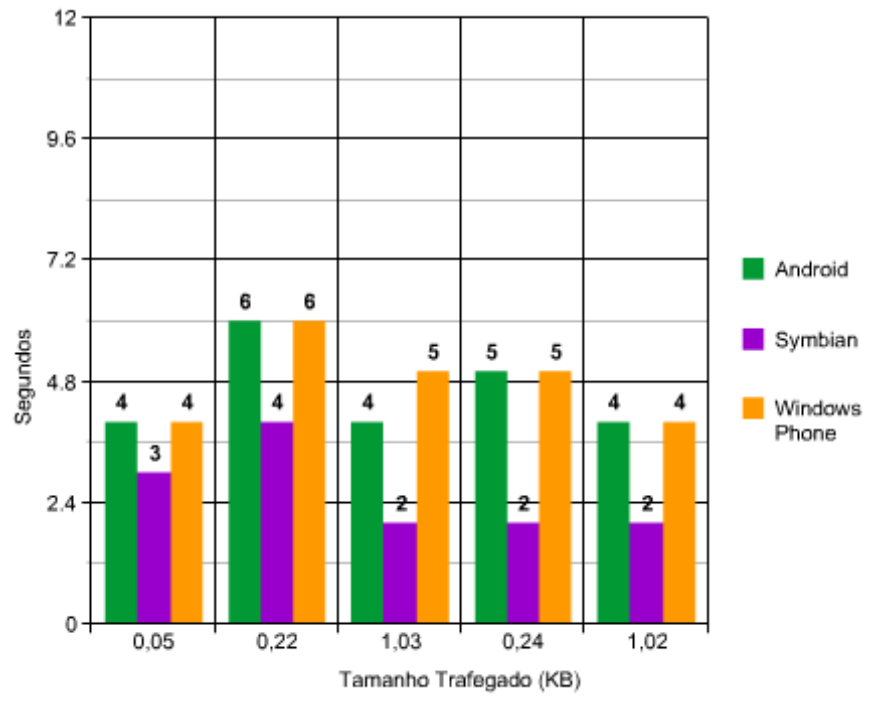

Figura 11: Gráfico de resultados do objetivo (b)

Conforme observado na comparação dos dispositivos avaliados, conclui-se que o dispositivo executando o Chronos Mobi em plataforma Symbian obteve melhor desempenho, justificando-se pelo fato de que nesta plataforma foram utilizadas somente tecnologias web. Enquanto nas demais plataformas, o Phonegap deve responsabilizar-se pela conversão da linguagem web para a linguagem nativa de cada plataforma agregando mais processamento, consequentemente maior tempo para execução.

\section{Conclusão}

Este artigo apresentou uma solução para o gerenciamento de projetos baseada no uso de dispositivos móveis, projetada para executar em diferentes plataformas e tipos de dispositivos. O Chronos Mobi proporciona maior eficiência e produtividade no gerenciamento de projetos, pois otimiza e agiliza o gerenciamento, apresentando telas adaptáveis de acordo com o perfil do stakeholder e o tipo de dispositivo móvel utilizado, além de exibir projetos e tarefas de acordo com o contexto no qual o usuário encontra-se.

Além disso, este artigo apresentou uma análise comparativa do Chronos Mobi executando em múltiplas plataformas, baseando-se no framework para desenvolvimento móvel Phonegap.

Conclui-se que a plataforma Symbian possui maior velocidade na renderização das telas da aplicação por suportar diretamente tecnologias web, descartando-se a conversão para a linguagem nativa. Conclui-se também que existe uma forte tendência na adoção de frameworks para desenvolvimento multiplataforma com o uso de tecnologias web.

Alguns trabalhos futuros planejados para a evolução do projeto Chronos Mobi são: (a) ampliar o escopo da avaliação para recursos adicionais, tais como GPS e acelerômetro; (b) realizar testes de carga e performance com uma massa de dados mais ampla; (c) explorar o uso de técnicas Scrum na execução das tarefas; (d) ampliar os recursos de segurança da aplicação. 


\section{Referências}

[1] APPCELERATOR. Framework Appcelerator. Disponível em: <http://www.appcelerator.com>. Acesso em: 03 nov. 2012.

[23] APPCELERATOR. Appcelerator. Disponível em: <http://www.appcelerator.com/thinkmobile/surveys>. Acesso em: 03 nov. 2012.

[3] APPLE. iOS Technology Overview, Apple Inc, $1^{\text {st }}$ edition, 2010.

[4] APPLE. Apple Member Center. Disponível em: <https://daw.apple.com/cgibin/WebObjects/DSAuthWeb.woa/wa/login?appIdKey=cdd9076ec329293eceabffdaf0aa8bb31 fac601 da0e97 f2f78bc379783c46096\&path=\%2F\%2Fmembercenter\%2Findex.action>. Acesso em: 03 nov. 2012.

[5] APPLE. Apple Developer. Disponível em: <https://developer.apple.com/programs/start/standard/create.php>. Acesso em: 03 nov. 2012.

[6] APP HUB. App Hub. Disponível em: <http://create.msdn.com/en-US/home/membership>. Acesso em: 03 nov. 2012.

[7] APP STORE. App Store. Disponível em: <http://apple.com/br/mac/app-store>. Acesso em: 03 nov. 2012.

[8] BABIN, S. Developing Software for Symbian OS, Wiley, 2th edition, 2007.

[9] BATISTA, M.; TAVARES, J.; BARCELOS, G.; FILIPPETTO, A.; BARBOSA, J. Chronos: A Model for Ubiquitous Project. In: International Conference on Applied Computing. Rio de Janeiro. v.1. p.1-3.

[10] CYGWIN. Cygwin. Disponível em: <http://www.cygwin.com/>. Acesso em: 03 nov. 2012.

[11] DEY, A. Understanding and Using Context. In: Personal and Ubiquitous Computing. v. 5. p. 1-4, 2001.

[12] DOTPROJECT. DotProject. Disponível em: <www.dotproject.net>. Acesso em: 03 nov. 2012.

[13] ECLIPSE. Eclipse IDE. Disponível em: <http://www.eclipse.org>. Acesso em: 03 nov. 2012.

[14] GOOGLE PLAY. Google Play. Disponível em: <https://play.google.com>. Acesso em: 03 nov. 2012.

[15] IBM. IBM. Disponível em: <https://www.ibm.com/developerworks/mydeveloperworks/blogs/752a690f8e93-4948-b7a3-c060117e8665/entry/pesquisa_para_onde_vai_o_desenvolvimento_movel?lang=en>. Acesso em: 03 nov. 2012.

[16] IDPH. IDPH. Disponível em: <http://www.idph.com.br/artigos/novaeducacao/mapasmentais.php>. Acesso em: 03 nov. 2012.

[17] IMASTERS. Desenvolvendo aplicativos para múltiplas plataformas. Disponível em: $<$ http://imasters.com.br/artigo/21010/mobile/desenvolvendo-aplicativos-moveis-para-multiplas-plataformas>. Acesso em: 03 nov. 2012.

[18] JORDAN, L. Project Management with dotProject, Packt Publishing, 1th edition, 2007.

[19] JQUERY. JQuery Mobile. Disponível em: <http://www.jquerymobile.com>. Acesso em: 03 nov. 2012.

[20] JSON. Json. Disponível em: <www.json.org>. Acesso em: 03 nov. 2012.

[21] LUNNY, A. Phonegap Beginner’s Guide, Packt Publishing, 1th edition, 2011.

Revista Brasileira de Computação Aplicada (ISSN 2176-6649), Passo Fundo, v. 5, n. 1, p. 84-97, abr. 201396 
[22] MICROSOFT. Microsoft. Disponível em: <http://www.microsoft.com/windowsphone/pt-br/>. Acesso em: 03 nov. 2012.

[23] PHONEGAP. Framework Phonegap. Disponível em: <http://phonegap.com>. Acesso em: 03 nov. 2012.

[24] PMBOK. PMBoK. Disponível em: <http://www.pmi.org/PMBOK-Guide-and-Standards.aspx>. Acesso em: 03 nov. 2012.

[25] PMI. A Guide to the Project Management Body of Knowledge (PMBoK), 4th edition, 2000.

[26] PMI. PMI Institute. Disponível em: <http://www.pmi.org>. Acesso em: 03 nov. 2012.

[27] PROTOTYPE. Prototype. Disponível em: <http://www.prototypejs.org>. Acesso em: 03 nov. 2012.

[28] RHOMOBILE. Framework Rhomobile. Disponível em: <http://www.rhomobile.com>. Acesso em: 03 nov. 2012.

[29] SATYANARAYANAN, M. Pervasive computing: Vision and challenges. IEEE Personal Communications. v.4. p.1-7, 2001.

[30] SCHWABER, K.; WOOLF, B. What's so eXtreme about doing things right. International Conference on Object-Oriented Programming, Systems, Languages and Applications, pp. 26-30, 2003.

[31] VISUAL STUDIO. Visual Studio. Disponível em: <http://www.microsoft.com/visualstudio/pt-br/homeprodutos>. Acesso em: 03 nov. 2012.

[32] W3SCHOOLS. Portal W3Schools. Disponível em: <http://www.w3schools.com>. Acesso em: 03 nov. 2012.

[33] WEISER, M. The computer for the Twenty-First Century. Scientific American. v.1. p.1-2, 1991.

[34] XCODE. XCode. Disponível em: <https://developer.apple.com/xcode/>. Acesso em: 03 nov. 2012. 